\title{
Prácticas de los abogados en formación en los consultorios jurídicos ${ }^{1}$
}

Practices of lawyers in training in legal consulting offices

Julián Osorio Valencia ${ }^{2}$

Recepción: 8 de agosto de 2016 Aprobación: 20 de enero de 2017

\section{Resumen}

Este artículo complementa y amplía la tesis de la débil pertinencia de los programas de derecho - por la desarticulación entre planes de estudio y prácticas jurídicascon la tesis de la institucionalización del diálogo pedagógico contextual, en las áreas del conocimiento jurídico. La proposición se fundamenta en la hermenéutica filosófica de la comprensión de la experiencia humana de mundo como dialogismo contextual, de Gadamer. Su aplicación a la comprensión de la experiencia de la formación profesional, situada en los contextos problemáticos de las prácticas jurídicas, gesta, además de la contextualización de los planes de estudio, ambientes de aprendizaje interdisciplinar en la comprensión de estas prácticas.

1 Artículo producto del proyecto de investigación Las prácticas de los abogados en formación en los consultorios jurídicos. Complementa y amplía la investigación gestionada por la Asociación Colombiana de Facultades de Derecho (Acofade) y el Ministerio de Justicia y del Derecho.

2 Estudiante de Doctorado en Filosofía, Universidad del Valle; Magister en Filosofía y Magister en Derecho Administrativo; Filósofo; profesor de la Facultad de Derecho, Universidad Libre, seccional Pereira, y miembro del grupo de investigación Derecho, Estado y Sociedad. Correo electrónico: josorio@unilibrepereira.edu.co 
Palabras clave: formación profesional, dialogismo contextual, pedagogía, desarrollo profesional docente.

\section{SUMMARY}

The focus of the paper is to complement and to extend the assertion about a low relevance of the programs of study in law faculties (due to the lack of coordination between the curriculums and the professional practices). The problem is analyzed from the thesis of the institutionalization of the contextual educational dialogue in the areas of legal knowledge. This proposition is based on the philosophical hermeneutics of the comprehension of the human experience about the world as a contextual dialogism of Gadamer. Its application in understanding the experience of a vocational training into a problematic context of the professional practices enables the learning in interdisciplinary environments, in addition to the contextualization of the programs of study.

Keywords: Vocational training, contextual dialogismo, pedagogy, teacher professional development.

\section{INTRODUCCIÓN}

La contextualización de la función social del derecho en la construcción de una cultura de paz en la vivencia del postacuerdo está mediada por la eficacia social de la gestión del conocimiento jurídico para profundizar la modernidad social, como formación de ciudadanía y sujetos de derechos; la democracia participativa, como deliberación pública en derecho, y la democratización de las prácticas económicas, con sentido de la sostenibilidad ambiental, como negociación de intereses en conflicto siguiendo procedimientos en derecho.

La idoneidad de esta mediación demanda ampliar la comprensión del problema de la formación profesional integral de los nuevos juristas y la actualización de los juristas en ejercicio. De una parte, como ciudadanos profesionales con capacidades y actitudes necesarias para el fortalecimiento de las capacidades institucionales de la 
sociedad y el Estado, a partir de la dinamización de un diálogo interinstitucional, transinstitucional e intercultural orientado al entendimiento. Y de la otra, de profesionales disciplinares capaces de incidir en los proyectos del desarrollo humano, social, cultural, académico, científico, económico y ambiental, y en el desarrollo de las capacidades de la sociedad para la exigibilidad de sus derechos.

A nuestro juicio, un primer paso para ensanchar la comprensión del problema de la formación profesional integral de los juristas en formación y la actualización de los juristas en servicio se dirige a examinar la tradición educativa de los juristas en la que estamos insertos. Aquí se busca reconocer la pertinencia y relevancia de lo que hacemos frente a las exigencias formativas de los juristas para la construcción de una cultura de paz en la vivencia de la democracia participativa para la endogenización de un desarrollo equitativo y sostenible.

El alcance del ascenso en el conocimiento propiciado por la tradición educativa jurídica para construir una cultura de paz se examina en la reflexión de sus prácticas. Por esta razón, es necesario recomponer el estado del arte en la institucionalización de un diálogo abierto interdisciplinar, transdisciplinar e intercultural en los espacios organizacionales de los docentes. No solo para reconstruir sus percepciones, de camino a una fundamentación epistemológica de la práctica de la enseñanza- aprendizaje de las ciencias jurídicas y la formación de competencias profesionales, en relación con el proceder establecido en las políticas estatales de formación profesional; sino también para mostrar que la ineficacia social en la pretensión de lograr la calidad de la formación jurídica integral exclusivamente a partir de lo declarado unilateralmente en los mandamientos normativos, se explica por no considerar la experiencia docente.

En relación con la valoración de la pertinencia de las prácticas con el comportamiento normado, desde nuestro punto de vista, la universidad aún no logra dimensionar la importancia de trascender la preocupante formalización de los espacios organizacionales de los docentes, institucionalizando el diálogo pedagógico en ellos, para superar el academicismo monodisciplinar, por fuera de los contextos problemáticos de las prácticas profesionales (o con alusiones coyunturales a estas). Es decir, aún no orienta en los espacios organizacionales de los docentes un diálogo interdisciplinar, transdisciplinar e intercultural en el conocimiento académico de las prácticas jurídicas para su innovación como ambientes de aprendizaje en contexto. 
En relación con la segunda situación y como consecuencia del confort propio en la formalización de los espacios organizacionales de los docentes, la universidad aún no conciencia que desde la sola advocación normativa a la gestión del PEI para la formación integral, acompańada del requerimiento de la alta titulación, sin tener en cuenta la experiencia docente, no se desarrollan efectivamente las capacidades institucionales para que la docencia universitaria correlacione la unidad entre la práctica profesional y su saber disciplinar respectivo, unida a la formación ciudadana de profesionales sujetos de derechos.

El Estado, la sociedad y las instituciones educativas privadas han supuesto equivocadamente que la intencionalidad del ideal estatal de la formación integral por competencias se garantiza con solo construir plantas físicas, dotarlas y nombrar o contratar los docentes.

En línea con este supuesto, la sociedad, el Estado y la academia han sobreentendido equivocadamente que los docentes pueden situar, desde su alta titularidad, sus conocimientos disciplinares en los contextos del acontecer facultativo de los profesionales en formación. Colateralmente, desde un reconocimiento trivial al conocimiento pedagógico, la academia ha asumido que con solo informar sobre las teorías pedagógicas, los docentes pueden inferir el saber específico de la mediación didáctica, para dinamizar el diálogo académico interdisciplinar, transdisciplinar e intercultural en el aula y en los grupos de investigación, situado en el contexto de las prácticas profesionales.

En suma, la institucionalidad colombiana aspira a que los docentes desarrollen la formación integral desde la universalización de la lógica inductiva del "comuníquese y cúmplase", sin el explíquese in situ. Se está ante un enciclopedismo que perdió de vista considerar que, en la comprensión y aplicación del comportamiento atribuible al mandamiento legal de la formación profesional por competencias, es necesario tener en cuenta la experiencia de los docentes, en la implementación de esa ordenación.

El emplazamiento en este estudio de la perspectiva que considera la experiencia docente, para la institucionalización de la política de la formación profesional integral, se apoya en la hermenéutica de la comprensión de la experiencia acontecida desde el dialogismo contextual en Gadamer. Nuestra hipótesis de labor es que a través de este diálogo los docentes comprenden sus dificultades y advierten sus potencialidades 
para gestar experiencias de gestión de la calidad (pertinencia y relevancia) de la formación profesional integral.

En el caso de la formación profesional de los nuevos juristas, la trascendencia del olvido por el conocimiento de la experiencia docente en sus desempeños institucionales, de aula y extra-aula, mueve a emplazar su reflexión en los espacios organizacionales de las áreas del conocimiento jurídico (hoy preocupantemente formalizados); a fin de dinamizar un diálogo interdisciplinar e intercultural que sitúe los planes de estudio en los escenarios de las prácticas profesionales.

En atención a este vacío de gestión del conocimiento, para instituir un diálogo pedagógico en los contextos de la formación jurídica, la presente investigación se propuso complementar y ampliar la tesis de los investigadores de la Asociación Colombiana de Facultades de Derecho (Acofade) y Ministerio de Justicia y del Derecho de la desarticulación de los planes de estudio de los contextos problemáticos de las prácticas jurídicas. Para ello, partió del examen del alcance en la comprensión del saber específico de la pedagogía, expuesto en los resultados de la investigación sobre las prácticas de los abogados en formación en los consultorios jurídicos.

En el informe de Acofade y el Ministerio de Justicia y del Derecho, el diagnóstico descrito se acompañó de la producción de unos lineamientos técnicos para resignificar la administración pedagógica de la enseñanza-aprendizaje del derecho. En estos lineamientos se invita a examinar la pregunta por el cómo instituir "estrategias pedagógicas y didácticas que articulen el aprendizaje del derecho con las necesidades del entorno" (p. 33). Sin duda, este reconocimiento al déficit de gestión del conocimiento pedagógico sobre las prácticas formativas de aula y extraaula en los programas de derecho, actualiza el ignorado examen por el vacío de pedagogía realizado por la "Misión de sabios" (1994) para todo el sistema educativo.

La pregunta de Acofade y MinJusticia, y de la Misión de sabios por el cómo instituir el diálogo pedagógico en los programas de derecho permitió formular como hipótesis de labor que el concepto de hermenéutica filosófica de la comprensión de la experiencia humana de mundo como dialogismo contextual en Gadamer, aplicado al ámbito de la experiencia humana de la formación jurídica, facilita a directivos y 
docentes complementar y ensanchar la comprensión del problema de la institucionalización de la formación profesional integral en contexto.

A partir de esta hipótesis, se formuló la siguiente pregunta de investigación: ¿¿De qué manera, la hermenéutica filosófica de la comprensión de la experiencia humana del mundo como diálogo, en Hans Georg Gadamer, aplicada a la experiencia docente de la formación jurídica en el contexto de sus prácticas, complementa y ensancha el modo como el Estado y los programas de derecho comprenden y aplican la política de la formación profesional integral alrededor de las prácticas?

El hipotético reconocimiento de que la hermenéutica filosófica de la comprensión de la experiencia humana de mundo como dialogismo contextual en Gadamer (2005), aplicado a la experiencia formativa, permite ver de otra manera, y de un modo más abarcante, la praxis de la formación jurídica en contexto, se apoya en la noción gadameriana de formación y de experiencia formativa. Para Gadamer, la formación se advierte como "aquello en lo cual y a través de lo cual uno se forma" (2005, p.40). Siendo "aquello" un ambiente dialógico orientado al entendimiento, que tiene presente los juicios y prejuicios de los profesionales en formación, con los que se entra en una conversación académica en el aula; no para juzgarlos sino para complementarlos, ensancharlos y fusionarlos con los horizontes de sentido expuestos en el aula por los docentes.

En línea con el concepto de formación, la comprensión de la noción de experiencia formativa es esencial también para complementar y ensanchar las percepciones puestas en juego, por parte de la institucionalidad rectora de la educación y de los directivos docentes y docentes de los programas de derecho. De este modo, se facilita la interpretación y aplicación de la normatividad reguladora de la transformación de los escenarios de práctica en ambientes de aprendizaje, de camino a su conversión en epicentros de la formación profesional.

A nuestro juicio, la apropiación de la hermenéutica gadameriana y de las nociones de formación y experiencia formativa, en el marco del concepto de formación jurídica integral, en la base de la política de formación integral por competencias, orienta la comprensión y aplicación del dialogismo contextual en la correlación de la interdisciplinariedad e interculturalidad con los problemas de las prácticas jurídicas. 
Para hacer comprensible este planteamiento se estimó pertinente, en primera instancia, reconstruir los aspectos generales de la tradición interpretativa privilegiada por los investigadores de Acofade y MinJusticia. En línea con el diálogo aperturado con esta tradición y con la concienciación de la importancia sustancial de la reconstrucción de los saberes docentes, en un segundo momento se relacionan los elementos esenciales que determinan la concepción de la hermenéutica filosófica de la comprensión de la experiencia docente como diálogo contextual. Finalmente, o en tercer lugar, se fundamenta la necesidad de institucionalizar el estatus de cientificidad de la pedagogía. La institucionalización de este estatus posibilita el ensanchamiento de la actual política de desarrollo profesional docente, para el fortalecimiento de las capacidades institucionales en la armonización de la práctica profesional y su saber disciplinar respectivo.

La perspectiva metodológica acogida para la realización de la presente investigación es de corte hermenéutico filosófico. Los esfuerzos se concentraron en la comprensión de la noción de práctica jurídica instalada, desde la interpretación del sentido de la hermenéutica de la comprensión de Gadamer, aplicada a la experiencia docente como diálogo, en perspectiva a resignificarla, desde lo fundamentado en la actual política de formación integral de los nuevos juristas.

\section{LA TRADICIÓN INTERPRETATIVA PRIVILEGIADA EN LA INVESTIGACIÓN SOBRE LAS PRÁCTICAS JURÍDICAS}

El diagnóstico sobre las prácticas de los abogados en formación en los consultorios jurídicos, expuestos en la investigación de Acofade-MinJusticia (Molina, et al., 2014), identifica como causa — de la débil pertinencia de los programas de derecho con las demandas sociales de formación jurídica - "la obsolescencia de la normatividad reguladora de las prácticas” (Ley 583 de 2000 que modificó el Decreto 196 de 1971). Allí se afirma que el desarrollo normativo vigente no determina convertir en ambientes de aprendizaje las situaciones que presenta la ciudadanía en los consultorios jurídicos ni tampoco "las tendencias del derecho internacional" ni las "del nuevo orden constitucional”.

El vacío de conocimiento concienciado en la investigación sobre las prácticas en los consultorios jurídicos explica por qué los docentes organizados en las áreas del 
conocimiento jurídico y en los grupos de investigación no usan "los casos más emblemáticos y de mayor impacto, que se conocen en los consultorios jurídicos y en las aulas, ni ingresarlos a los planes de estudio, ni fundamentar las líneas o grupos de investigación” (Molina, et al. 2014, p.18). De manera extensiva, la concienciación de este vacío de conocimiento también permite explicar por qué no es posible gestionar la formación profesional integral.

Los investigadores de Acofade y MinJusticia también observan que el efecto de la débil gestión del conocimiento para dinamizar el proceso de la formación profesional integral es la reedición de la tradición transmisionista. Esta situación la recrean los investigadores de Acofade y MinJusticia cuando registran que "no se brinda a los estudiantes las posibilidades de desarrollar habilidades para el litigio, ni se inculca el rol de los abogados en la sociedad, ni se promueve la implementación y desarrollo de métodos de análisis críticos y de litigios estratégicos más allá de la transmisión de un enciclopedismo "bancario"” (Amsterdam, 2003, p. 7).

Las percepciones reconstruidas de los profesores de derecho muestran que las debilidades del sistema de prácticas jurídicas en Colombia "se encuentran en el modelo tradicional de enseñanza del derecho implementando en Colombia”. No solo porque "la formación práctica se imparta a partir de los dos últimos años de la carrera profesional", sino ante todo porque el proceso educativo se centra en la rutinaria enseñanza escolástica "de modelos teóricos y memorísticos, desligados de la práctica como forma de aprendizaje" (Molina, et al., 2014, p. 13).

La percepción de los docentes sobre la desarticulación entre teorías disciplinares, prácticas profesionales y necesidades de formación del entorno externo también la tienen los estudiantes de derecho participantes en la investigación aquí analizada.

Una cantidad considerable de estudiantes considera que dichas prácticas son aburridas, impuestas y no le aportan mayor formación en su ejercicio profesional. Además, manifiestan que su plan de estudios no es coherente con la práctica, que sus conocimientos son insuficientes para las distintas áreas y que, en algunas, como en la penal y laboral, falta mayor coherencia entre teoría y práctica, mayor competencia litigiosa y una mejor actualización temática. Igualmente, la mayoría de estudiantes considera 
que no todas las áreas del derecho están comprendidas en la oferta y que necesitan una mayor posibilidad de prácticas (Molina, et al., 2014, p.18).

Las percepciones de profesores y estudiantes reconstruidas por los investigadores de Acofade y MinJusticia, mostrando la falta de coherencia entre planes de estudio y prácticas en los consultorios jurídicos confirma la tesis de la Misión de sabios, de la ausencia de un conocimiento propio para el ejercicio profesional de la enseñanza.

La aserción de la Misión, puesta en el ámbito de la formación integral de los juristas que la sociedad demanda, establece la necesidad de implementar un proceso de gestión del conocimiento que ponga a tono los programas de derecho con la innovación introducida en la reforma educativa de una formación profesional integral. La finalidad de gestionar la pertinencia del conocimiento disciplinar con los contextos de las prácticas profesionales busca encuentro con la determinación de acciones que innoven, de un lado, la tradición transmisionista de información y conocimientos jurídicos, situando los currículos en los contextos problemáticos de las prácticas profesionales. Y del otro, que transforme las prácticas, tanto de la enseñanza-aprendizaje disciplinar del derecho como de la formación y desarrollo de las competencias profesionales, generales y específicas, declaradas en los proyectos educativos de los programas de derecho.

Frente a este têlos las percepciones de los investigadores de Acofade y MinJusticia requieren la actualización de la norma reguladora de las prácticas jurídicas y demandan la gestión pedagógica de un plan de estudios pertinente con las demandas formativas de los contextos profesionales de los juristas. Empero, frente a lo demandado procede distinguir la naturaleza del conocimiento que se requiere para administrar la articulación del currículo al contexto, en perspectiva a transformar las prácticas jurídicas en ambientes de aprendizaje.

La naturaleza del conocimiento para instituir la reforma reguladora de las prácticas jurídicas, que busca producir unos lineamientos que orienten la contextualización de los currículos con las demandas de formación en los escenarios de práctica profesional es diferente de la naturaleza del conocimiento que se requiere para la transformación de las prácticas de aula. 
En el primer caso, se trata de un conocimiento técnico-administrativo que tiene por objeto producir una regla reguladora y una pauta orientadora de la gestión de la articulación de un programa académico a un contexto ${ }^{3}$. En el segundo, se trata de un conocimiento propio de la pedagogía, cuyo objeto se ocupa del proceso de la formación en contexto. El conocimiento técnico administrativo es auxiliar del conocimiento pedagógico. El primero se ocupa del qué hacer para administrar la contextualización del currículo. Mientras que el pedagógico se ocupa del cómo diseñar y realizar la medicación didáctica, para dinamizar el proceso de la formación profesional integral en contexto.

En apoyo de esta distinción procede recurrir a Gadamer cuando muestra que "el resultado de la formación no se produce al modo de los objetos técnicos, sino que surge del proceso interior de la formación y conformación y se encuentra por ello en un constante desarrollo y progresión" (p. 40). El currículo es un medio y como tal no puede dar cuenta del proceso interior de la formación y conformación de sentidos. En un plano inmanente el currículo es un instrumento que permite integrar en el plan de estudio los contenidos y temas de las áreas del conocimiento con las demandas de formación contextual, supuestamente priorizadas desde los diagnósticos contextuales en el PEI e identificadas como núcleos problemáticos. Empero, un diseño curricular así concebido si bien encauza el alejamiento de la escuela de los ambientes socioculturales y productivos, su aplicación no da cuenta del problema del saber orientar el favorecimiento en la vida universitaria la formación y conformación del desarrollo profesional integral en las aulas y en los escenarios institucionales y extrainstitucionales. El objetivo de la formación integral no se determina, a priori, desde el saber situar pertinentemente el plan de estudio con las demandas de formación del entorno externo y las expectativas formativas de los profesionales en formación. Para dinamizar la formación y conformación de sentidos es necesaria la apropiación inter e intrainstitucional tanto del enfoque del desarrollo integral por competencias

3 La gestión del conocimiento para la lectura de las tendencias problemáticas de los distintos niveles contextuales (internacional, nacional, local), la priorización de problemas de acuerdo con la naturaleza académica del programa y de sus énfasis, y la inferencia de necesidades de formación, y su definición como núcleos problemáticos de los proyectos educativos de los programas es de tipo técnico-administrativo. Distinto de la naturaleza pedagógica del conocimiento con el cual se gestionan las dinámicas dialógicas docente-docente en los espacios organizativos de las áreas del conocimiento, preparadoras del diálogo interdisciplinar, transdisciplinar e intercultural docentedicente en el aula. 
(establecido en la política educativa) como del enfoque y del modelo pedagógico orientador de las mediaciones metodológicas y didácticas, prescrito en el proyecto educativo institucional.

La asimilación del enfoque del desarrollo y pedagógico así como del modelo pedagógico es la condición subjetiva necesaria no solo para implementar un diseño curricular pertinente, sino también, y de manera fundamental, para dar cuenta de la realización del objetivo de la formación en la relación docente-dicente en el aula y en los espacios institucionales y territoriales. Es a través del proceso de asimilación del enfoque del desarrollo y del modelo y enfoque pedagógico como se podrá dar cuenta de las condiciones que necesitan los docentes para dinamizar la experiencia del proceso de la formación profesional integral en contexto.

Situada la experiencia de la formación como experiencia dialógica que dinamiza aquello en lo cual y a través de lo cual uno se forma, en la visión gadameriana de que educar es educarse, la perspectiva autopoiética del objeto de la formación, entendido como aprender a saber autoformarse, "no conoce objetivos que le sean exteriores" (p. 40). Puesto que se trata del proceso interior e histórico de la formación y conformación de sentidos con los cuales opera, en el mundo de la vida profesional, el que se forma. Por esta razón, Gadamer mantiene que "la formación no puede ser un verdadero objetivo; (este) no puede ser querido como tal si no es en la temática reflexiva del educador" (p.40).

Puesta en contexto la tesis gadameriana, el camino de la construcción del objetivo de la formación profesional solo puede emprenderse en el dialogismo contextual de los docentes, organizados en sus áreas de conocimiento. La normatividad reguladora de las prácticas jurídicas y de su gestión curricular es el medio a través del cual se organiza el modo de administrar la articulación de los programas de derecho a los contextos de las prácticas profesionales. Por esta razón, la normatividad como medio no puede dar cuenta del objetivo de la formación. Este, como nota Gadamer, no puede ser querido como tal sino en la dinámica reflexiva del educador.

Así las cosas, el diálogo interdisciplinar, transdisciplinar e intercultural contextual requiere como condición de la intersubjetividad docente la apropiación institucional del enfoque y modelo pedagógico determinado en el proyecto educativo del pro- 
grama académico. Es en este escenario donde se construyen acuerdos para diseñar e implementar estrategias pedagógicas y mediaciones metodológicas y didácticas que dinamicen el proceso de enseñanza-aprendizaje del conocimiento jurídico y de formación y desarrollo de competencias profesionales generales y específicas, situadas en el contexto de los problemas del desarrollo y de la constitucionalización del Estado y del derecho.

El diálogo pedagógico contextual docente es el camino para corresponder a las recomendaciones de Molina (et al., 2014) cuando, al apremiar el inicio temprano de las prácticas jurídicas, demanda que se las implemente "como una matriz de transversalidad que permee todo el plan de estudios, lejos del componente asignaturista, de tal manera, que permita articular más la teoría con la práctica” (p.55). En otras palabras, la conversión de los escenarios de la prácticas profesionales en "laboratorio adecuado para proponer estrategias pedagógicas y didácticas que articulen el aprendizaje del derecho con las necesidades del entorno" (p. 56) solo puede ser producto del diálogo de saberes docente reflexionando pedagógicamente la conversión de las prácticas en ambientes de aprendizaje.

La tesis de la articulación pedagógica del aprendizaje con las necesidades del entorno afirmada por los investigadores de Acofade y MinJusticia, acogida y ampliada aquí con la tesis de la hermenéutica filosófica del dialogismo contextual docente, se propone mostrar que el camino de la construcción del saber situar el plan de estudios en los contextos situacionales de los escenarios de las prácticas profesionales y del saber dinamizar el aprender a saber autoformarse, está mediada por la institucionalización del dialogismo contextual docente. Desde luego, hay que admitir que la institucionalización de la reflexión del relacionamiento de las prácticas jurídicas con los planes de estudio y del saber gestar innovadoramente las mediaciones didácticas transformadoras de las prácticas escolásticas, requiere institucionalizar la investigación pedagógica para acompańar este relacionamiento y transformación, y no solo para teorizarlo.

Ahora bien, en el entretanto del empoderamiento de la investigación pedagógica institucional, para la sistematización del conocimiento producido como teorización de la reflexión de la práctica profesional docente, la rica tradición de la enseñanza del derecho por profesionales disciplinares requiere de acompañamiento experto externo in situ. Tanto para la gestión académica de los componentes pedagógico, metodoló- 
gico y didáctico, con los cuales desplegar ejercicios de dinamización del diálogo de saberes entre docentes y estudiantes sobre la experiencia vivida en el desempeño de las prácticas profesionales como para teorizar el conocimiento producido en el proceso de este acompañamiento experto.

El conocimiento práctico así producido es el que permite reelaborar el saber específico de la pedagogía. Puesto ahora al servicio tanto del saber situar dialógicamente los planes de estudio en las situaciones reales que se presentan en los escenarios de las prácticas profesionales como al servicio del saber diseñar, implementar y evaluar las mediaciones metodológicas y didácticas que dinamizan la formación y conformación de sentidos que dinamizan aquello en lo cual y a través de lo cual uno se forma.

La consideración al miramiento de la tradición educativa en derecho por parte de los investigadores de Acofade y MinJusticia permitió caer en cuenta de que el diseño curricular del transmisionismo de información y conocimientos por fuera de los contextos problemáticos de las prácticas profesionales no forma en la acción y menos para la acción profesional. Se está ante una valoración que verifica la inocuidad de la pretensión instalada de direccionar la formación profesional sin la iluminación del diálogo pedagógico. No porque no se reconozca la pedagogía, sino porque se la trivializa como información teorética escindida de la formación investigativa de las prácticas. Es decir, no se la asume como ciencia de la obra de arte de la formación. Esto se aprecia al constatar que en los requerimientos de selección docente solo se considera la formación disciplinar de cualquier profesional, con el barniz de un curso de pedagogía.

Si la idoneidad del jurista, economista, médico, enfermero, sociólogo, psicólogo, trabajador social impone el dominio de la disciplina respectiva, porque sin ella no hay autoridad en el desempeño, con mayor razón, es necesario ennoblecer la disciplina pedagógica, que ilumina el idóneo ejercicio de la profesión que se ocupa del objeto de la formación (ciudadana y profesional). A la reflexión pedagógica no se la puede seguir minimizando creyendo que para formar en cualquier profesión basta el conocimiento disciplinar respectivo y no que sea necesario, también, el saber específico del arte de la formación, es decir, la disciplina pedagógica.

Por esta razón, se honra de los investigadores de Acofade y del Ministerio de Justicia y del Derecho (Molina, et al., 2014) la intuición pedagógica que entraña su preo- 
cupación por re-significar el sentido de la planeación curricular, de camino a revisar sus contenidos en el contexto de las prácticas jurídicas de los programas de derecho. No tanto para saber "a ciencia cierta si desde estos escenarios se está haciendo lo necesario para la buena formación de los abogados y el buen funcionamiento de la justicia en Colombia” (Molina, et al., 2014, p.19); puesto que factualmente sabemos que no. Sino para pensar pedagógicamente el movimiento del giro copernicano de un currículo que parte del concepto y versa todo el tiempo sobre el concepto, con coloquiales alegorías a la realidad, hacia un currículo que parta de la experiencia de aprendizaje, en la acción formadora en los contextos de las prácticas jurídicas y en su tradición histórico epistémica, para llegar a los conceptos, teorías y doctrinas, a partir de las cuales desarrollar las competencias profesionales y la formación de actitudes para el diálogo interhumano, sobre todo hoy, cuando nos disponemos a la construcción de una cultura de paz en la vivencia del postacuerdo.

Sin duda, es un adelanto que los investigadores de Acofade y MinJusticia (Molina, et al., 2014) vean la necesidad de la reorganización curricular. Pero si esta se piensa en perspectiva a la formación profesional integral requiere la institucionalización de la pedagogía y la investigación pedagógica en el contexto universitario. Es a través de la gestión del conocimiento pedagógico como se alcanza la progresión y desarrollo de un dialogismo contextual entre los docentes, que empodere el movimiento del giro curricular advertido, a dinamizar en el aula.

La ponderación por los actores intervinientes sobre la importancia sustantiva de la planeación curricular (por ejemplo, del plan de estudios en el contexto de las prácticas jurídicas) induce a que se administre pedagógicamente el comportamiento imputable a los factores de calidad de los programas de derecho (académico, investigativo y de proyección social). Es decir, interpela la institucionalización de la reflexión pedagógica, hoy sustituida por la logística instrumental de las TIC y por el formalismo que caracteriza la implementación de unos procesos de autoevaluación institucional sin que medie la investigación pedagógica de la pertinencia de las funciones sustantivas de la academia con las demandas de formación, de gestión del conocimiento y de proyección social identificadas en los escenarios de práctica.

La generalización de la práctica del diálogo pedagógico, orientado al entendimiento, entre los docentes organizados en las áreas del conocimiento y en los grupos de inves- 
tigación, con los docentes que asesoran las prácticas, con el apoyo de la investigación pedagógica, posibilita ampliar la comprensión de la tradición de nuestras prácticas planificadoras, implementadoras y evaluadoras de la formación profesional integral en contexto.

La reflexión del problema de la formación de la persona humana en general y su formación profesional en particular ponen de presente que el alcance práctico de la comprensión de "aquello en lo cual y a través de lo cual uno se forma" (Gadamer, 2005, p.18) se da en el contexto de la reflexión pedagógica de la formación y conformación de sentidos, a través de una mediación didáctica que convierta en ambientes y experiencias de aprendizaje los contextos problemáticos del mundo de la vida en general y profesional específica.

Administrativamente, esto significa que la planeación curricular debe ocuparse de situar los contenidos disciplinares de los planes de estudio en la diversidad de escenarios problemáticos del mundo de la vida profesional jurídica. Pedagógicamente quiere decir que el dialogismo contextual debe ocuparse del problema de la mediación didáctica, desde la implementación de una estrategia de apropiación institucional del enfoque del desarrollo de la política educativa y del componente pedagógico en el proyecto educativo del programa.

Sin embargo, como se viene advirtiendo, este saber situar curricularmente los planes de estudio e innovar la mediación didáctica para el dialogismo contextual en el aula es el menos desarrollado por nuestra tradición educativa. Quizás por la trivialización de la pedagogía como ciencia de la formación básica y profesional. Se trata de una ligereza que, al soslayar la formación investigativa pedagógica, permitió que la sola acreditación del título profesional disciplinar habilite para desempeñarse profesional de la docencia sin el conocimiento del saber pedagógico históricamente constituido El cual es sustituido ahora en forma minimizada, con algunas teorías descontextualizadas del proceso formativo, que no logran desarrollar la competencia pedagógica de los profesionales de la docencia jurídica como está probado de hecho.

Esta minimización de la pedagogía como ciencia en la gestión curricular (académica, investigativa y de proyección social) y en la mediación didáctica, se advierte también cuando se la asume en un sentido instrumental. En la investigación interpretada se 
trae a colación una cita donde "Se concibe la pedagogía como un proceso de aprendizaje para transformar, con métodos dinámicos y participativos. En este esquema, el maestro juega un papel activo, creador, investigador y experimentador; y el estudiante se desempeńa como un sujeto con competencias previas de creatividad, reflexión, intereses de conocimiento propios” (Montoya, 2012, p.133).

Si bien, esta concepción, rebasa la práctica curricular del transmisionismo escolástico, es instrumental. Reduce la pedagogía a una técnica de clase activa que, a pesar de su importancia, no deja de ser un suplemento del objeto de la formación y conformación de sentidos, el cual debe ser estudiado como un asunto complejo, propio de la ciencia pedagógica. La experiencia de la clase activa debe ser reflexionada como una experiencia docente puesta en perspectiva a gestionar la mediación didáctica interdisciplinar, transdisciplinar e intercultural en el contexto de las prácticas jurídicas. Desde esta consideración, lo fundamental es no perder de vista la reflexión colectiva de la experiencia docente sobre sus prácticas de aula, entre ellas la clase activa. El objeto de la pedagogía como reflexión de la aplicación de la hermenéutica filosófica de la experiencia humana del mundo como diálogo en Gadamer, aplicada a la experiencia del aprendizaje en los contextos de las prácticas jurídicas es el camino a dilucidar.

La elucidación de la experiencia docente como diálogo no se puede desplegar desde los saberes declarados en la política, en la normatividad, en las filosofías de la educación, ni desde los saberes de las disciplinas generales y específicas de las profesiones, menos desde el procedimiento universal establecido en los estándares de competencias. Se trata de una clarificación alcanzable paulatinamente en el dialogismo contextual de los profesionales de la educación con competencia pedagógica.

Por esta razón, el objetivo de la formación y conformación de sentidos, es decir, de aquello en lo cual y a través de lo cual uno se forma, requiere del saber específico de la pedagogía producido a través de la investigación pedagógica sobre las prácticas de enseñanza-aprendizaje y desarrollo de competencias en contexto. La gestión del conocimiento producido a través de una investigación pedagógica que acompañe in situ el proceso de la formación y desarrollo de la competencia pedagógica de los docentes en servicio se erige en el factor dinamizador de la promoción de actitudes dialógicas sobre mediación didáctica propicia para la dinamización de las experien- 
cias de aprendizaje de la diversidad de saberes disciplinares, a situar curricularmente en los contextos de las prácticas profesionales convenidas.

De manera general, de la tradición interpretativa de los investigadores de Acofade y MinJusticia sobre las prácticas jurídicas en los programas de derecho se pondera que advierte la importancia de planes de estudios situados en los escenarios de práctica. De hecho, acoge una perspectiva que intuye la necesidad de la reflexión pedagógica, pero sin reconocer la importancia sustancial de desplegarla en la vida institucional como dialogismo contextual, con rigor de ciencia. Por lo pronto, en los planes de desarrollo profesional docente, se debe dar cabida a la incorporación del acompańamiento pedagógico experto externo para aprender a desarrollar la gestión del conocimiento para una mediación didáctica que dinamice la formación profesional de los nuevos juristas, situando curricularmente los planes de estudios en la diversidad contextual de las demandas de formación inferidas de los problemas en el desempeño de la práctica profesional jurídica.

Instituir la pedagogía como saber específico que dinamice curricularmente el "juego" del arte de la formación y conformación de sentidos como interacción dialógica docente-dicente-texto en contexto es la necesidad por satisfacer desde los planes de desarrollo profesional pedagógico docente. El reto de reconocer el valor esencial de la pedagogía como dialogismo contextual científico fue el que ponderó la Misión de Sabios en 1994, cuando recomendó crear un "Instituto de Pedagogía" que le dé estatus al saber docente, puesto que este va más allá del saber disciplinar con alta titulación.

\section{LA HERMENÉUTICA FILOSÓFICA DEL DIALOGISMO CONTEXTUAL DE GADAMER}

La tesis de la comprensión de la experiencia del ser humano en el mundo como dialogismo contextual en Gadamer, le lleva a explicitar cómo es posible la comprensión de una experiencia que forma y conforma el modo de ser del hombre en el mundo.

Gadamer inicia problematizando la inconveniencia del método inductivo para comprender la experiencia del hombre en su estadía mundana. La impredecibilidad de las decisiones que emergen desde el ejercicio del libre albedrío, en la praxis de los 
comportamientos relacionales del hombre consigo mismo, el otro y su entorno físico biótico, imposibilita identificar regularidades que permitan formular predicciones a los comportamientos relacionales humanos. Como se trata de una verdad que "no queda correctamente aprehendida si se la mide según el patrón del conocimiento progresivo de leyes" (Gadamer, Verdad y Método I, p. 32), Gadamer plantea el problema de la concreción histórica de la verdad de esta experiencia.

Apoyándose en la preestructura de la comprensión del modo de ser del hombre en el mundo (Heidegger), Gadamer explica que si "el ser es tiempo", la comprensión del modo de ser del ser-en-el-mundo interpretando su experiencia mundana se desvela como tiempo histórico del "estar-ahí" en el mundo siendo intérprete de su propia experiencia.

La analítica temporal del "estar ahí" conformando un modo de ser interpretativo de su experiencia en la estadía mundana, lleva a Gadamer a comprobar que el discernimiento de esta experiencia se da desde la comprensión de los horizontes de sentidos sedimentados en la temporalidad del devenir, con los que los seres humanos operan en su estadía mundana.

De acuerdo con Gadamer, la comprensión de esta experiencia interpretativa no busca fundamentar un modelo conceptual del modo de ser interpretativo, sino asegurar la cientificidad de la comprensión de un modo de ser interpretativo, que por ser histórico y finito, sedimenta, junto a los sentidos asentados históricamente, con los cuales se opera en la mundanidad, los prejuicios de la cultura y del lenguaje, que dinamizan a su vez las ocurrencias previas de los intérpretes. A esta sedimentación de sentidos y prejuicios Gadamer la llama "historia efectual".

La comprensión de la experiencia de la interpretación del mundo de la vida desde la historia efectual, a juicio de Gadamer, "no es uno de los modos del comportamiento del sujeto, sino el modo de ser propio del estar ahí, en-el-mundo" (p. 12), siendo intérprete, "en una progresión constante e interminable" (p. 44).

Si se acepta la tesis de que hay un ser en el mundo que interpreta lo que hace en ese mundo para comprenderlo desde su historia efectual, emerge el problema de cómo fijar científicamente el resultado de la pluralidad de interpretaciones puestas en juego 
a partir de la diversidad de historias efectuales. Gadamer, apoyándose en Heidegger, plantea que la cientificidad en la interpretación de la temática o asuntividad objeto de comprensión se asegura desde la cosa misma. Para ello, Gadamer propone relacionarla con su predeterminación histórica o tradición que la sustenta. De este modo, la cientificidad o interpretación correcta del tema o del asunto objeto de interpretación hace referencia a la necesidad de no perder de vista que toda interpretación, por estar mediada por prejuicios y hábitos del pensar, requiere depurarse en el diálogo con la tradición que sustenta el asunto objeto de interpretación.

¿Qué se puede aprender de la tradición? En la tradición se encuentran las viejas verdades, la verdadera sabiduría de la vida, "que tiene que ver más con el ideal práctico de la phrónesis (prudencia del saber práctico) que con el teórico de la sophia". El saber práctico que determina el sentido común (sensus communis) es el sentido que "funda la comunidad y orienta su voluntad" (p. 50). "No es la generalidad abstracta de la razón, sino la generalidad concreta (del saber práctico) que representa la comunidad de un grupo, de un pueblo, de una nación o del género humano en su conjunto" (p. 50).

Como generalidad concreta, el saber práctico, la phrónesis, es una forma de saber "orientada hacia la situación concreta". Esto significa que "se sustrae al concepto racional de saber" (p. 51). Es decir, opone al saber por principios generales el saber de lo concreto que permite dilucidar la dirección correcta. Ahora bien, el esclarecimiento de la dirección correcta presupone una orientación ética de la voluntad, de camino a distinguir lo conveniente de lo inconveniente para la vida en comunidad.

De esta manera, la formación del sensus communis de una comunidad se revela como la experiencia de la interrelación del saber concreto con la ética, para la conformación de "un sentido que se adquiere a través de la comunidad de vida y (que) es determinado por las ordenaciones y objetivos de ésta” (p. 52). Como la determinación normativa de la vida en comunidad "mantiene el valor y el sentido de las propias tradiciones de vida estatal y social” (p. 52), en la fundamentación del sentido común de la vida en comunidad, la historia de sus tradiciones representa "una fuente de verdad muy distinta de la razón teórica” (p. 53). 
En el entendido de que el conocimiento de la tradición no busca el conocimiento progresivo de leyes, lo que esta enseńa de la experiencia de la vida en común es que la vida en comunión es experiencia de la finitud humana. Al no existir un alguien que lo sepa todo se reconocen "los límites de toda previsión y la inseguridad de todo plan” (p. 427). Por esta razón, al hacerse consciente los límites del poder hacer se gesta la autoconciencia de una razón planificadora, la cual, de acuerdo con Gadamer, privilegia la experiencia por referencia a sus resultados. Para él, estos suelen ser esencialmente negativos, puesto que refieren rupturas con generalidades típicas que han de ser destipificadas o con generalizaciones falsas que han de ser refutadas. Esto significa que no habíamos visto correctamente las cosas y que es ahora cuando por fin nos damos cuenta de cómo son.

La reflexión dialógica de la negatividad de la experiencia hace posible "acceder a un mejor saber, no solo sobre él sino sobre aquello que antes se creía saber”. Es un diálogo a través del cual alcanzar lo que Gadamer llama una generalidad. Es decir, un saber no concluyente. Como no es la generalidad del concepto ni de la ciencia, sino una generalidad que posibilita "actualizar las observaciones individuales", a esta interpretación dialógica de la experiencia Gadamer le da el nombre de dialéctica. A través de este dialogismo con la negatividad de la experiencia se "da apertura no solo a nuevas experiencias sino también a correcciones de errores" (p. 427). En Gadamer, el concepto de experiencia adquiere un momento cualitativamente nuevo. La experiencia no se ahorra a nadie y solo se adquiere a través de decepciones.

En el contexto de comunidades educativas, la formación del sensus communis, a través de la experimentación de su conformación, es decisivo para su transformación en comunidades académicas. El arte de encontrar argumentos que contribuyan a la formación de un sentido común que determine lo convincente a la luz de la dirección correcta en la dinamización del mandamiento normativo de la formación integral no se posibilita per se desde el mandamiento normativo. Se requiere conformar comunidades de diálogo y su reconocimiento por el Estado como alteridad válida para identificar los factores potenciadores de las transformaciones educativas en la base de la política de calidad estatal.

La superación de la enajenación de la conciencia docente para actualizar los conocimientos disciplinares y los estándares de competencias profesionales en los contextos 
de los programas de derecho, se puede abordar desde la hermenéutica del dialogismo contextual de Gadamer (2011). Su institucionalización, a manera de complementación del actual modo de significar del Estado, facilita no solo el ensanchamiento de los conocimientos disciplinares y los estándares de competencias propuestos, sino asumirlos, no como verdad universal, sino como "un otro", objeto de los diversos modos de significación pedagógica de los docentes, para ser tenidos efectivamente en cuenta por el Estado en perspectiva real para ajustar su discurso. Esto implica avanzar en la modernización de un Estado que posibilite construir acuerdos en torno a los mejores argumentos, siguiendo procedimientos en derecho.

Desde luego, objetarán algunos que el Estado permite la libre expresión docente. Sí. Pero no se trata de la generalidad que declara que a nadie se le niega que exprese su punto de vista. En efecto, no se niega, pero no se tienen definidos procedimientos en derecho que tengan en cuenta los mejores argumentos para una transferencia del nuevo conocimiento que actualice las normas. Específicamente, para ampliar el alcance del concepto de formación profesional integral.

La perspectiva del dialogismo contextual docente, que se ocupa de pensar, gestar y aplicar la mediación pedagógica y didáctica propicia para situar pertinentemente la formación integral en los entornos regional y nacional, como no se activa desde lo declarado en las normas, requiere del Estado la recreación de condiciones para la vivencia del diálogo interpretativo de directivos y docentes, en la experiencia de la contextualización de la normativa y política de la formación integral.

Esta interacción dialógica contextual dinamizaría la construcción de un sensus communis que formaría y conformaría un sentido general de la formación integral del nuevo jurista, capaz de hacer distinciones frente al discurso del Estado, para establecer el objetivo de la formación, a validar en el acontecer de la formación en contexto. Este camino del dialogismo contextual entre docentes, dicentes y agentes del Estado, para conformar un sentido comunitario de la formación, a partir de la política de formación profesional por competencias concederá ampliar el alcance del concepto de formación integral en contexto, como diálogo interhumano orientado a la comprensión del acaecer vital de los abogados en formación. 
Se trata de una perspectiva que demanda reflexionar el tema del desarrollo profesional docente que, a nuestro juicio, no posibilita la alta titulación postgradual, sino la concienciación de la importancia radical de institucionalizar una política de formación pedagógica que active auténticamente el desarrollo profesional de los profesionales de la educación en todos sus niveles y profesiones.

\section{INSTITUCIONALIZACIÓN DEL ESTATUS DE CIENTIFICIDAD DE LA PEDAGOGÍA}

La disposición del propósito de dar apertura, en el sistema educativo colombiano, a los cambios más significativos puestos en acción en otras partes del mundo, fue prevista hace 22 años por la Comisión de los Sabios (Aldana et al., 1996) constituida por el presidente César Gaviria.

Esta misión diagnosticó que el sistema educativo colombiano se caracterizaba por la fragmentación y dispersión del conocimiento, por no interpretar las demandas de formación de los contextos y por centrarse en memorizar aspectos secundarios. Para superar este estado, recomendó provocar en los docentes el desarrollo del pensamiento pedagógico. Para ello avizoró que "[...] es urgente iniciar la experiencia de profesionales universitarios de la pedagogía que luego se especialicen en un área o nivel de la educación, y también en diseñar programas que permitan a los profesionales de cada una de las disciplinas adquirir la formación pedagógica seria que el país y los alumnos necesitan" (Aldana et al., 1996, p.63). Lo avizorado por los sabios profundizó la solución al recomendar:

Crear un Instituto Nacional Superior de Pedagogía de nivel universitario, que se extenderá a una red de institutos análogos en las regiones, en los que se realice investigación educativa en programas profesionales de cinco ańos, con elevados requisitos de ingreso, becas para los mejores estudiantes de cada departamento que quieran dedicarse a esta profesión. Transformar las mejores de las actuales facultades de educación en institutos superiores de pedagogía regionales, o en centros de formación especializada en la docencia de un nivel o área del saber, para la formación pedagógica de los egresados de otras profesiones que quieran dedicarse a la enseńanza. Se trata de llegar a la excelencia 
en la pedagogía, y luego profundizar en una de las disciplinas, o de llegar a la excelencia en las disciplinas respectivas, y luego profundizar en la pedagogía, sin aceptar ligeros barnices de una u otra. Solo así se recobrará paulatinamente el estatus del docente como profesional e intelectual de alto nivel. Renovar la formación continuada de los docentes [...] centrar la formación continuada y el perfeccionamiento docente en la pedagogía” [...] (Aldana et al., 1996, pp.107-109).

Durante estos 22 años, si bien se advierten avances en una reforma jurídica y una estructura discursiva disciplinar que sirve de soporte para implementar una oferta educativa pertinente con las demandas constitucionales de una formación social participativa y económica competitiva, es notable que el componente de la formación para la investigación pedagógica sigue aplazado en el tiempo. Por esta causa, en lugar de incidir en la transformación de las prácticas sociales y productivas se conservan y promueven relaciones interhumanas determinadas por la histórica realidad de la guerra: negación de la alteridad, desconfianza, olvido del otro, falta de carácter, ausencia de sentimiento de vida espiritual, insensibilidad con las obligaciones para con uno mismo, violencia generalizada, incapacidad para el diálogo entre interlocutores con diferentes expectativas de vida y pobreza.

Este panorama desalentador lleva a que en el imaginario social se perciba que en la educación nada ha cambiado a pesar de los esfuerzos estatales de dar apertura al cambio y de realizar la reforma jurídica y discursiva para implementarlo. Y sobre todo, de haber aclarado que el problema de la calidad de la educación se resuelve instituyendo el estatus de cientificidad de la disciplina pedagógica para transformar las prácticas educativas de la tradición escolástica. Responsabilidad que no es del Estado sino de la academia universitaria.

Así como el médico cura la enfermedad con el conocimiento anatómico y biológico del cuerpo humano y el abogado resuelve los pleitos con el conocimiento de las leyes, el docente necesita del saber pedagógico para resolver los asuntos relacionados con la formación. Pero el Ministerio de Justicia y del Derecho no forma a los abogados; a estos los forman los programas de derecho, el MinJusticia proporciona los medios. De la misma forma, al Ministerio de Educación no le corresponde configurar la ciencia pedagógica, más bien proporciona medios para que la academia la construya 
y forme los docentes que el país requiere para llenar los vacíos identificados en las recomendaciones de los sabios y dar pasos firmes en las transformaciones de las prácticas educativas instaladas.

La tarea de comprender y ensanchar el horizonte de exigencias formativas en los abogados en formación, requiere, de parte de la academia, desarrollar más conciencia política frente a la importancia decisiva de la cualificación de la educación por vía de la pedagogía como ciencia de la obra de arte de la formación y del enseñar a aprender a educarse así mismo, para participar de manera idónea y democrática en la gestión de la juridificación del desarrollo humano, social, cultural, académico, científico, económico y ambiental, local e internacional.

Se trata de retomar la recomendación de la Misión de sabios para crear un instituto de pedagogía que inicie el proceso de llenar la ausencia de saber pedagógico en el contexto de todos los niveles educativos, reconociendo y sistematizando los esfuerzos de los docentes por innovar. Entre los factores que justifican su creación, además de las recomendaciones de los sabios, está también la premonición, en este sentido, del profesor Leonardo Velásquez cuando dice:

Insistimos que los cambios en las prácticas de formación no se derivan de su existencia jurídica [...] Los cambios educativos aclamados por la infancia, la adolescencia, la juventud, la sociedad y el Estado resultarán de los procesos investigativos de los maestros organizados en centros académicos, ligados estrechamente con las prácticas pedagógicas. Procesos que implicarían reflexiones, interpretaciones, narraciones, ensayos, artículos, monografías, innovaciones y transformaciones" (Velásquez, 2003, p.82).

El auspicio y estímulo a la investigación entre los docentes recomendada por la comisión de sabios se diluyó en una política de promoción de la iniciativa individual de cada docente a partir de premios. Mientras la investigación pedagógica no se integre como factor de desarrollo en la educación superior, en los regímenes estatutarios de la docencia se queda en letra muerta y la tradición se mantiene. La transferencia de sus innovaciones es el medio dinamizador del dialogismo contextual docente.

Es necesario apreciar que los modelos de calidad en los países que ocupan el primer lugar en las evaluaciones internacionales como Singapur y Finlandia, el Estado parte 
del esfuerzo de sus instituciones universitarias y de los maestros consagrados por vocación a la experimentación e investigación pedagógica. Estas experiencias internacionales marcan la ruta para Colombia. El Estado debe reconocer, valorar, auspiciar, patrocinar y adquirir ofertas de estas alianzas. Desconocer su propio patrimonio de saber, exportando mecánicamente experiencias, es lo que se hizo durante los siglos XIX y XX, con resultados desastrosos registrados en el informe Colombia al Filo de la Oportunidad, de la Comisión de sabios.

No solo es necesario reconocer lo propio sino también actualizar las recomendaciones de los sabios. Frente a ello, el investigador colombiano, con asiento en la universidad de Harvard, Rodolfo Llinás, presidiendo el encuentro científico en la Universidad Tecnológica de Pereira en agosto de 2015, dijo que las recomendaciones elaboradas por esta Comisión, "quedaron convertidas en letra muerta". Ante la persistencia de una práctica educativa de mala calidad reclamó:

¿Cómo es posible que no se hayan hecho una gran cantidad de sugerencias que pudieron haber mejorado la situación de la educación en Colombia? [...] se sigue enseñando en escuelas, colegios y universidades con los mismos parámetros que en su momento se criticaron y eso es una bestialidad [...] se enseña sin asegurarse de que el educando entienda lo aprendido [...] (Llinás, 2015).

En la educación colombiana todo permanece igual, nada ha cambiado, porque la reforma jurídica y discursiva llevada hasta ahora no ha llegado hasta la mente de los docentes ni a las estructuras curriculares de los diferentes niveles de la educación por la falta de esa institución de pedagogía, que construya saber pedagógico desde el saber implícito en las prácticas de formación que a diario realizan los docentes y que ellos mismos deben sistematizar, en comunidades académicas, con el apoyo de expertos en investigación pedagógica.

En el marco de la comprensión de la experiencia formativa en Gadamer (2011) para el ensanchamiento de la actual política de formación profesional integral a través del instituto de pedagogía, es muy importante tener una legislación educativa con los principales postulados de la pedagogía moderna y contemporánea.

En la especificidad que nos ocupa, implementar los estándares de competencias profesionales en las ciencias jurídicas, en el proceso de formación profesional integral, 
presupone administrar la aplicación de la política y de la normatividad reguladora de las prácticas jurídicas, en el marco del proceso de autoevaluación institucional que implementa el Consejo Nacional de Acreditación. Esto significa, como muy bien lo señalaron los investigadores de Acofade y MinJusticia (Molina et al., 2011) actualizar dicha normatividad. Para ello, es preciso instituir una situación de diálogo abierto entre directivos docentes, administrativos, docentes y estudiantes de los programas de derecho con los actores institucionales de los escenarios de la práctica jurídica.

Esta apertura al diálogo en contexto, no solo facilita la apropiación de un espacio de comprensión de la política de formación integral que privilegia elementos éticos y ontológicos diversos en la reflexión pedagógica de la experiencia de su institucionalización a través de las prácticas jurídicas, sino que, de acuerdo con Gadamer (2011), constituye una de las actitudes ineludibles para llevar a cabo procesos formativos de autocomprensión con el otro, esencial en la concreción del propósito constitucional de una formación social participativa, pluralista y democrática, así como en el de la constitucionalización del Estado y del derecho, siguiendo procedimientos en derecho.

En concordancia con lo dispuesto anteriormente, se considera oportuno resaltar lo dispuesto por los profesores Tirado y Blanco, quienes opinan que:

El ser humano persigue la inmortalidad mediante el mejoramiento de la expectativa de vida y persigue un mayor conocimiento mediante la estimulación de los sentidos, el uso de tecnologías para la recolección y memorización de información; así como el uso de sustancias — nootrópicos — para incrementar la concentración o la productividad de los individuos" (Tirado \& Blanco, 2016, p.92).

\section{Resultados}

El diálogo de los investigadores de Acofade y MinJusticia con la tradición educativa del transmisionismo de información y conocimientos por fuera del acontecer profesional de los abogados en formación constató su débil eficacia social para gestionar el conocimiento de los problemas de la formación profesional integral en el contexto de sus prácticas. 
Es necesario conocer la tradición de una oferta de titulación que privilegia sin más el ascenso a la cima de la erudición teorética, distante del conocimiento de las prácticas jurídicas que demandan los problemas de constitucionalización del Estado y del derecho para el nuevo orden social. Para ello se debe instituir la gestión del conocimiento pedagógico en aras de dinamizar el dialogismo contextual docente en la comprensión de la formación y conformación de sentidos en el contexto de las prácticas jurídicas, a través de los cuales se autoforman los profesionales en formación. Los conocimientos gestionados sobre la relación docente-dicente, texto en contexto son elaboraciones desde las cuales emplazar un dialogismo contextual que construya acuerdos metodológicos y didácticos que dinamicen el proceso de la autoformación.

La complementación y ampliación de la afirmación de los investigadores de Acofade y MinJusticia de la débil pertinencia de los programas de derecho, por la desarticulación entre planes de estudio y prácticas jurídicas, con la tesis de la institucionalización del diálogo pedagógico en las áreas del conocimiento jurídico, apoyándose en la hermenéutica filosófica de la comprensión de la experiencia humana de mundo como dialogismo contextual de Gadamer, permite no solo dilucidar el camino de la comprensión de la experiencia de la formación profesional situada en los contextos problemáticos de las prácticas jurídicas, sino también advertir que este diálogo pedagógico gesta, además de la contextualización de los planes de estudio, la dinamización de ambientes de aprendizaje interdisciplinar en la comprensión de estas prácticas.

Por esta razón, el emplazamiento de un diálogo pedagógico que reconstruya la experiencia de aprendizaje en el contexto de las prácticas profesionales, determina la necesidad de instituir la pedagogía como la disciplina que posibilita la comprensión de la experiencia docente, para aprender, no solo a saber situar los planes de estudio en los contextos de las prácticas profesionales, sino también a dinamizar el diálogo interdisciplinar en las aulas, para la comprensión de los problemas jurídicos y sociojurídicos en los contextos de las prácticas jurídicas.

Finalmente, la institucionalización de este dialogismo contextual requiere, más que programación de capacitaciones docentes, de la implementación de planes de desarrollo profesional docente, que empoderen el diálogo de su tradición educativa con la política nacional de formación profesional por competencias y de ciencia, tecnología e innovación, para la formación y conformación de un sensus communis en torno al 
cómo hacer pertinente la formación profesional integral instalada y la gestión del conocimiento en los ámbitos de interés del Estado, de la sociedad y de la propia academia.

\section{Conclusiones}

Los hallazgos de esta investigación clarifican la urgente necesidad de institucionalizar el dialogismo pedagógico sobre las prácticas profesionales en los espacios organizativos de los docentes (áreas del conocimiento) a fin de situar los planes de estudio como agentes transformadores de las prácticas jurídicas priorizadas para convertirlas en ambientes de aprendizaje interdisciplinar en las aulas.

Este diálogo de saberes pedagógicos debe extenderse a los procesos de autoevaluación institucional de la gestión de la investigación sobre la pertinencia curricular de los programas de derecho.

Los comités de ética de la investigación deben incorporar en la evaluación de la investigación el componente de transferencia de estos conocimientos al currículo, verificando el nivel de contextualización de los estándares nacionales e internacionales de pertinencia formativa.

El Estado debe asumir la responsabilidad de actualizar la normatividad reguladora del servicio social de los consultorios jurídicos y centros de conciliación, en perspectiva a su conversión en ambientes de aprendizaje.

Para institucionalizar la reflexión pedagógica en la educación superior urge que la academia dialogue acerca de la naturaleza del instituto de pedagogía.

\section{REFERENCIAS}

Aldana E., Chaparro L., García G., Gutiérrez R., Llinás R., Palacios M., Patarroyo M., Posada E., Restrepo A., Vasco C. (1996). Colombia al filo de la oportunidad. Informe de la Misión de Sabios. Presidencia de la República, Consejería Presidencial para el Desarrollo Institucional, Colciencias. Bogotá: Tercer Mundo Editores. 
Amsterdam, A. (2003). "Clinical Legal Education”. En A. Hurder (ed.). Clinical Anthology, Readings for Live-Client Clinics. Ohio: Anderson's Law School Publications.

Colombia. Congreso de la República (2000). Ley 583: modifica el Decreto 196 de 1971. Bogotá Colombia. Recuperado de: http://www.mineducacion.gov.co/1621/articles-105018_ archivo_pdf.pdf

Colombia. Congreso de la Republica (2008). Decreto Ley 1188. Bogotá Colombia. Recuperado de: http://www.cna.gov.co/1741/articles-186370_LEY_1188.pdf

Colombia. Congreso de la Republica (2009). Ley 1286. Recuperado de: http://www.alcaldiabogota.gov.co/sisjur/normas/Norma1.jsp?i=34850

Colombia. Ministerio de Educación Nacional, MEN (1994). Ley General de Educación 115. Bogotá, Colombia.

Colombia. Ministerio de Educación Nacional, MEN (2006). Propuesta de Lineamientos para la Formación por Competencias en Educación Superior. Bogotá, Colombia. Recuperado de: http://www.mineducacion.gov.co/1621/w3-article-340021.html

Gadamer, H. G. (2005). Verdad y Método. Salamanca: Ed. Sígueme.

Gadamer, H. G. (2011). Educar es Educarse. Nuevas corrientes intelectuales. 6 (1), 92-109.

Habermas, J. (2008). Facticidady Validez. Madrid: Trotta.

Hegel, F. (1993) Fenomenología del Espiritu. México: FCE.

Llinás, R. (2015). "Se perdió el trabajo de la Comisión de sabios". Pereira: Periódico La tarde. 15 de agosto de 2015. Recuperado de: http://www.latarde.com/noticias/ pereira/155849-se-perdio-el-trabajo-de-la-comision-de-sabios-llinas

Molina, C.M.; Carreño, M.T.; Sayas, R.; Montoya, C.; Herreño, D.; Alarcón, Y. \& Álvarez, J.R. (2014). Diagnóstico y lineamientos técnicos para los distintos escenarios de la práctica juridica de los programas de derecho de las instituciones de educación superior en Colombia. Medellín Colombia: Acofade, Ministerio de Justicia y del Derecho, UniRemigton. Recuperado de: http://www.uniremington.edu.co/presencial/images/stories/es/fondoeditorial/diagnosticoylineamientos.pdf

Montoya, M. (2012). Los consultorios jurídicos como metodología del aprendizaje del derecho. Medellín: Corporación Universitaria Remington. 
Tirado, M \& Blanco, C (2016). "Bioética y transhumanidad: hacia una aproximación al consumo de sustancias nootrópicas en el campo académico". En Bioética y Docencia, pp.91-119. Bogotá: Editorial Ibáńez. Recuperado de: http://porticus.usantotomas.edu. co/bitstream/11634/1527/1/Bioetica\%20y\%20Docencia\%20\%282a.\%20diagramacion\%29.pdf

Velásquez, L. (2003). Educación, docencia y formación humana en Colombia. Pereira: Gráficas Buda. 